\title{
Thyroid cancer in the Era of COVID-19
}

\author{
Anabella Smulever $\mathbb{D}^{1} \cdot$ Erika Abelleira $\mathbb{I}^{1} \cdot$ Fernanda Bueno $\mathbb{D}^{1} \cdot$ Fabián Pitoia $\mathbb{D}^{1}$
}

Received: 5 June 2020 / Accepted: 27 July 2020

(c) Springer Science+Business Media, LLC, part of Springer Nature 2020

\begin{abstract}
The recent coronavirus infectious disease (COVID-19) caused by severe acute respiratory syndrome coronavirus 2 (SARSCoV-2) is placing health systems in serious challenges worldwide. Shocking statistics each day has prompted the World Health Organization to officially declare the COVID-19 outbreak as a pandemic in March 2020. Preliminary studies have shown increased mortality in patients with solid cancers and infection by SARS-CoV-2. Until now, the evidence on the behavior of COVID-19 in patients with a history of thyroid cancer remains scarce, and most of the recommendations given are based on common sense. Therefore, in this viewpoint, we present a brief review of several challenges we are frequently facing during this pandemic and a series of recommendations based on what we have implemented in our clinical practice at a university hospital currently mostly dedicated to COVID-19.
\end{abstract}

Keywords Thyroid cancer $\cdot$ COVID- $19 \cdot$ Coronavirus $\cdot$ Cancer $\cdot$ Infectious disease

\section{Introduction}

The recent coronavirus infectious disease (COVID-19), caused by severe acute respiratory syndrome coronavirus 2 (SARS-CoV-2), is placing health systems in serious challenges worldwide. Shocking statistics each day has prompted the World Health Organization to officially declare the COVID-19 outbreak as a pandemic. By June 28th 2020, the rapid virus dissemination has accounted for about 9,843,073 confirmed cases and 495,760 deaths worldwide. Argentina contributed with 55,343 confirmed cases and 1192 fatal victims related to virus local circulation (https://www.who.int/emergencies/diseases/novel-corona virus-2019). Current reports have linked COVID-19-related mortality on the prominent impact of multi-organ dysfunction syndrome. This is a consequence of dysregulated immune responses given by increased cytokine levels (IL-6, IL-10, and TNF $\alpha$ ), lymphopenia (in CD4+ and CD8+ $\mathrm{T}$ cells), and decreased expression of IFN $\gamma$ in $\mathrm{CD} 4+\mathrm{T}$ cells [1]. This phenomenon was called the "cytokine storm" by some authors and was associated with disease severity and

Fabián Pitoia

fpitoia@intramed.net

1 Division of Endocrinology, Hospital de Clínicas, University of Buenos Aires, Buenos Aires, Argentina poor outcomes [2]. On the other hand, in 2018 there were 18 million new cases of cancer worldwide (https://www. who.int/cancer/PRGlobocanFinal.pdf?ua=1). Since this disease is considered an immunosuppressive status by itself, especially in some types of cancer, the medical community has become increasingly concerned about the potentially deleterious effects that COVID-19 infection may cause in these patients.

\section{COVID-19 and thyroid cancer}

There is scarce evidence about the impact of COVID-19 infection in patients with a history of thyroid cancer. In an effort to investigate a correlation between cancer and mortality and/or poor prognosis associated with COVID-19 infectious disease, recently two international collaborative clinical trials-the COVID-19 and Cancer Consortium (CCC19) [3] and Thoracic cancERs international coVid-19 cOLlaboraTion (TERAVOLT) [4]—were conducted to demonstrate this potential association. In the CCC19 study, data were collected from 928 patients with different types of active and/or previous malignancies (including 30 patients with head and neck carcinomas) and confirmed severe SARS-CoV2 infection from the United States, Canada, and Spain. Among the most significant findings, all-cause mortality was $13 \%$ within 30 days of COVID-19 diagnosis. Patients with progressing cancer were found to be 5.2 times more likely to die within 30 days compared with 
patients in remission or with no evidence of disease. Besides, stable, non-progressing cancer was associated with 1.79 times greater risk of death than no evidence of disease. Overall, $14 \%$ of all patients were admitted to the intensive care unit. Mechanical ventilation was required for $12 \%$ of all patients, and additional oxygen was required by $44 \%$ of patients [3]. In the TERAVOLT study, 400 thoracic cancer patients from 121 centers in 28 countries were evaluated. The data suggested a high mortality rate $(33 \%)$ and the main risk factors associated were age ( $>65$ years), comorbidities, ECOG performance status $>1$, steroids more than $10 \mathrm{mg} /$ day, and chemotherapy [4].

Although these studies show the first experiences related to COVID-19 in patients with cancer, probably the heterogeneous types of cancer, stages, and treatments would not allow drawing definitive conclusions than can be assumed in thyroid cancer patients. Surely this situation would be subject to future research with a longer follow-up. However, the limited current medical evidence suggests that there is no reason to believe that merely a history of thyroid cancer is enough to have an increased risk of developing COVID-19 infection or to evolve into a poor outcome. Nevertheless, some aspects of thyroid cancer treatment and follow-up should not be neglected.

\section{Levothyroxine therapy}

The cumulative evidence reveals that thyroid hormones can exert responses on diverse immune cells that affect several inflammation-related processes (such as chemotaxis, phagocytosis, generation of reactive oxygen species, and cytokine production) [5]. Thus, interactions between the endocrine and immune systems contribute to pathological conditions, including viral infections. Under these circumstances, treatment with levothyroxine could help to re-establish the normal physiological conditions in patients with definitive postoperative hypothyroidism. On the other hand, a recent study showed that hyperthyroidism was associated with an increased release of cytokines implicated in T-cell activation, such as IL-2 and IFN- $\gamma$ and pro-inflammatory cytokines IL-6 and TNF- $\alpha$, under inflammatory conditions. Seemingly, these changes did not relate to suppressive TSH levels, but rather to a markedly excess in serum thyroid hormone levels [6]. In conclusion, an appropriate thyroid hormone replacement therapy is suggested, to avoid immune dysfunction in patients with postoperative permanent hypothyroidism under nonsuppressive therapy.

\section{Thyroid fine needle aspiration biopsy and surgery}

The acute effects induced by surgery including immunosuppression should be considered for patients who recently underwent surgical treatment. The relationship between hypothalamic-pituitary-adrenal axis hyper-activation and post-operative state of stress, both physical and emotional, is well known. The persistence of increased cortisol levels, even for several days following surgery, enhances the expression of anti-inflammatory genes in leukocytes, which decreased production of many pro-inflammatory cytokines including IL-1, IL-2, IL-6, IL-11, IL-13, and TNF $\alpha$ as a compensatory mechanism in response to stress [7]. This negative immunomodulation is enhanced by catecholamine acute release by interacting with receptors present on the surface of immune cells [8]. Finally, anesthetics and analgesic agents used peri-operatively may have a direct toxic effect on immune system components, including opiates, among others [9].

However, there is no evidence to suggest that a patient with a recent history of thyroid surgery is at greater risk of infection.

Throughout these more than 100 days of the confinement period, only seven diagnostic fine-needle aspiration biopsies (FNAB) were performed in our institution on thyroid, lymph nodes, and metastatic samples. In addition, one anaplastic thyroid cancer was biopsied to confirm the diagnosis and to perform molecular testing. Besides, ten surgeries due to rapidly progressive or high-risk thyroid cancer were conducted, including total thyroidectomies, lymph node dissections, and brain metastasis exeresis. This implied a decrease in more than $98 \%$ of these procedures usually done in the same period in our clinical practice. Aditionally, in four patients, active surveillance was indicated as the first choice approach after a diagnosis of papillary thyroid carcinoma.

As it was already pointed by several authors, we also postponed diagnostic FNAB and surgeries in our area where we have a more restrictive quarantine phase, otherwise, we recommend maintaining a regular decisionmaking process [10]. In properly selected patients with suspected low-risk papillary thyroid carcinoma, active surveillance is strongly recommended as the first line of management [11]. Surgery should be done with no delay in patients with rapidly progressing tumors, suspicion of anaplastic, or poorly differentiated carcinomas.

\section{Radioactive iodine (RAI) therapy}

In recent decades, several studies have revealed suggestive data about bone marrow toxicity induced by cumulative radioactive iodine doses, leading to B-lymphocytes and helper T-lymphocytes depletion, among other immunity cells. These adverse events depend on the radiation dose and anatomical extension. However, most studies concluded that no clinically relevant immunosuppression is observed with conventional ${ }^{131}$ I low activity $[12,13]$. Although RAI therapy is still considered a tool for the 
treatment of patients with differentiated thyroid carcinoma, it is advisable to undertake efforts to select the proper candidates for this therapy. In this regard, we think that the administration of RAI should not be delayed in patients with a high risk of recurrence or with diagnosed and/or suspected distant metastases. However, we have postponed all radioiodine dose administration even considering those patients with an intermediate risk of recurrence. In this last group of patients, RAI may be deferred according to the dynamic risk assessment.

While conventional RAI dose therapy does not confer an immunosuppressive status, post-radiation isolation could difficult the support of patients at a potential COVID-19 infection. In this case, postponing RAI therapy is an option that should be individualized considering the risk groups.

\section{COVID-19 and follow-up of thyroid cancer patients}

The frequency and modality of surveillance for patients with thyroid cancer in the COVID-19 pandemic must be adapted according to the risk of recurrence and the response to treatment. For patients with low-risk papillary thyroid carcinoma under active surveillance, ultrasound monitoring may be deferred for 4-6 months from the agreed schedule over the first year and further 6 months after the second year.

Patients with an excellent, indeterminate, or biochemical incomplete response to treatment do not constitute a risk group for COVID-19 infection, and the frequency of structural disease is less than $4 \%, 15 \%$, and $20 \%$, respectively [14]. Consequently, annual monitoring may be postponed for a further 6-8 months. On the other hand, patients with non-progressive structural incomplete responses, especially those with lymph node involvement, active surveillance could be a valid alternative [15].

In our experience, 223 patients have been monitored since the COVID-19 pandemic began. Among them, 161 (72\%) had an excellent response (ER), consequently, it was decided to postpone the medical appointments through e-mail and/or telephone contacts. There were 55 (25\%) patients with indeterminate (IR) and biochemical incomplete (BIR) responses in which most of the controls were postponed, whereas in three patients $(5 \%)$ a new biochemical assessment was evaluated through telemedicine.

Finally, in the remaining $7(3 \%)$ patients with structural incomplete response, we decided to undergo active surveillance on lymph nodes metastasis with additional biochemical and ultrasound monitoring by office medical appointment.

\section{Multikinase inhibitors (MKIs) in advanced thyroid cancer}

Since VEGFR and c-kit are expressed in hematopoietic precursors playing a critical role in normal hematopoiesis and cellular growth, MKIs may induce bone marrow toxicity. MKIs-treated patients may have an increased risk of neutropenia and lymphopenia, with an incidence of up to $5 \%$ and up to $13 \%$, respectively [16-18]. These complications can potentially lead to opportunistic infections. The incidence of severe hematological adverse events may be enhanced by the bone marrow damage caused by previous high cumulative doses of radioiodine.

Regardless of speculative conclusions derived from the scarcity of published reports about patients with COVID-19 infectious disease being treated with MKI, it is strongly suggested that patients under systemic treatment be considered as a risk group for COVID-19 infection and poor outcomes [3, 4]. The optimal time to start MKIs therapy is always a challenge since the impact on disease prognosis and the impaired quality of life should be taking into consideration in the decision-making. In particular pandemic circumstances, for patients who are candidates to start MKIs treatment, it is extremely important to reconsider the risks and benefits of postponing the beginning of treatment with a personalized clinical evaluation. In our experience, among 31 advanced thyroid cancer patients, 22 (71\%) had disease progression since the onset of the COVID-19 pandemic [17 differentiated (DTC), 4 medullary (MTC), and one anaplastic thyroid carcinoma], and the remaining nine patients with stable disease continued MKIs therapy. The assessment of adverse events, biochemical, and imaging in this last group was carried out by telemedicine.

In patients with progressive disease, MKIs was prescribed. Of these 22 patients, seven $(32 \%)$ were unable to start MKI as a result of (1) not having health insurance coverage, (2) not performing preliminary biochemical tests due to temporary laboratory closings, and (3) the inability to obtain medications due to border restrictions. The remaining 15 patients started MKIs: first-line $(n=4,26 \%)$, second line $(n=10,67 \%)$, and one patient $(7 \%)$ could be included in a clinical trial.

As we believe that it is important to establish a close interaction with the medical staff through remote communication resources to avoid contact with the medical care system as much as possible, telemedicine controls were carried out in each patient with advanced disease (13,700 sessions: 11,000 and 2,700 in patients with progressive and stable disease, respectively). Office appointments for crucial therapeutic changes for patients with progressive disease (MKI initiation or changes for second or third-line treatments) were also carried out (66 sessions). In comparison with the same period before the pandemic onset, in this group of patients, our data indicate an increase in telemedicine controls by $147 \%$, while office appointments exhibited a decrease of $35.5 \%$.

A decision-making algorithm for patients with COVID-19 under MKIs treatment is described in Fig. 1. 
Fig. 1 Decision-making algorithm for patients with COVID-19 under MKIs treatment
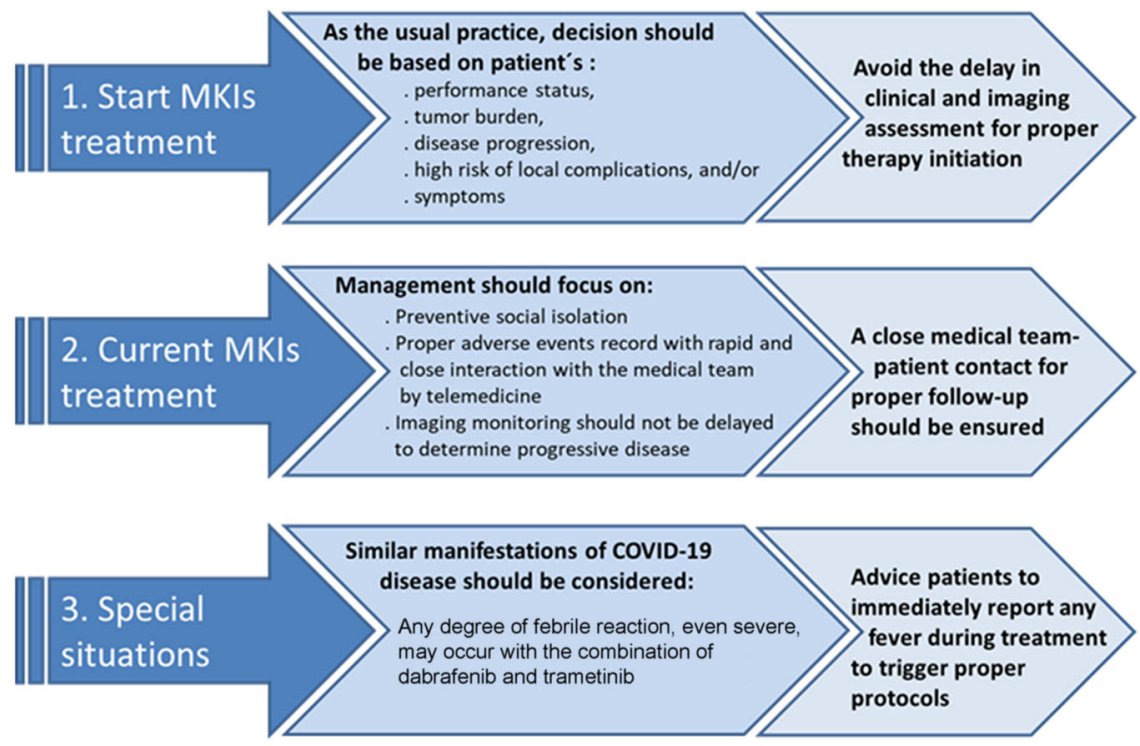

\section{External beam radiotherapy}

The effects on the lymphocyte count of external radiation therapy administered to treat non-lymphoid cancers have been extensively studied. In patients who received conventional external beam radiotherapy used to treat tumors originating from non-lymphoid tissues, an early reduction of the CD4+/ CD8+ ratio and impairment of antibody production have been observed. The resulting immunosuppressive condition may incur on a severe risk of infections [12].

Italy, which has been identified as one of the countries most affected by COVID-19, has been established practical indications for patients under radiotherapy. According to this statement, it was approved that if the patient has a fever or respiratory symptoms due to pre-existing morbidity, radiotherapy should be carried out as long as the use of a protective mask is guaranteed. Furthermore, patients may continue treatment only in selected cases, as if their general medical conditions are not compromised by COVID-19 infection, if the oncological condition requires an urgent continuation of radiotherapy, if it is permitted by local health authorities, and if they use of adequate disposable protective equipment is available [19]. In our latest practice, only two patients had to undergo external beam radiation therapy, performed on brain and pelvic metastasis. Considering the immunosuppression status acquired after radiotherapy, patients who have previously received external beam radiation to the neck should be considered as at increased risk of severe illness with COVID-19 and a personalized clinical evaluation is suggested.

The COVID-19 outbreak changed life as we have never imagined. If it was so for persons with no associated disorders, the concerns that we have observed in our patients with a past personal history of thyroid cancer, and the hundreds of questions we have answered regarding the higher risk for a worse outcome of this infection in several conditions associated with thyroid cancer patients prompted us to write this short editorial. As we stated, several different situations should be considered in thyroid cancer patients that may give them a higher risk, but definitive data for these affirmations are not yet available. Therefore, the recommendations provided here remain dynamic and should be tailored according to the doubling rates for confirmed COVID-19 cases as well as the epidemiological circumstances for each region.

Author contributions All authors contributed to the study conception and design. Material preparation, data collection and analysis were performed by A.S., E.A., F.B. and F.P. The first draft of the manuscript was written by Anabella Smulever and Fabian Pitoia and all authors commented on previous versions of the manuscript. All authors read and approved the final manuscript.

\section{Compliance with ethical standards}

Conflict of interest The authors declare that they have no conflict of interest.

Publisher's note Springer Nature remains neutral with regard to jurisdictional claims in published maps and institutional affiliations.

\section{References}

1. B. Diao, C. Wang, Y. Tan, X. Chen, Y. Liu, L. Ning, L. Chen, M. Li, Y. Liu, G. Wang, Z. Yuan, Z. Feng, Y. Zhang, Y. Wu, Y. Chen, Reduction and functional exhaustion of $\mathrm{T}$ cells in patients with coronavirus disease 2019 (COVID-19). Front. Immunol. 11, 827 (2020)

2. R. Channappanavar, S. Perlman, Pathogenic human coronavirus infections: causes and consequences of cytokine storm and immunopathology. Semin. Immunopathol. 39, 529-539 (2017) 
3. N.M. Kuderer, T.K. Choueiri, D.P. Shah, Y. Shyr, S.M. Rubinstein, D.R. Rivera, S. Shete, C.Y. Hsu, A. Desai, G. de Lima Lopes Jr, P. Grivas, C.A. Painter, S. Peters, M.A. Thompson, Z. Bakouny, G. Batist, T. Bekaii-Saab, M.A. Bilen, N. Bouganim, M. Bover Larroya, D. Castellano, S.A. Del Prete, D.B. Doroshow, EganP.C. Elkrief, A. Dimitrios Farmakiotis, D. Flora, M.D. Galsky, M.J. Glover, M.A. Griffiths, A.P. Gulati, S. Gupta, N. Hafez, T.R. Halfdanarson, J.E. Hawley, E. Hsu, A. Kasi, A.R. Khaki, C.A. Lemmon, C. Lewis, B. Logan, T. Masters, R.R. McKay, R.A. Mesa, A.K. Morgans, M.F. Mulcahy, O.A. Panagiotou, P. Peddi, N.A. Pennell, K. Reynolds, L.R. Rosen, R. Rosovsky, M. Salazar, A. Schmidt, S.A. Shah, J.A. Shaya, J. Steinharter, K.E. Stockerl-Goldstein, S. Subbiah, D.C. Vinhv, F.H. Wehbe, L.B. Weissmann, J. Tsu-Yu Wu, E. Wulff-Burchfield, Z. Xie, A. Yeh, P.P. Yu, A.I. Zhou, L. Zubiri, S. Mishra, G.H. Lyman, B.I. Rini, J.L. Warner, on behalf of the COVID-19 and Cancer Consortium: clinical impact of COVID-19 on patients with cancer (CCC19): a cohort study. Lancet 395, 1907-1918 (2020)

4. M.C. Garassino, J.G. Whisenant, L.C. Huang, A. Trama, V. Torri, F. Agustoni, J. Baena, G. Banna, R. Berardi, A.C. Bettini, E. Bria, M. Brighenti, J. Cadranel, A. De Toma, C. Chini, A. Cortellini, E. Felip, G. Finocchiaro, P. Garrido, C. Genova, R. Giusti, V. Gregorc, F. Grossi, F. Grosso, S. Intagliata, N. La Verde, S.V. Liu, J. Mazieres, E. Mercadante, O. Michielin, G. Minuti, D. MoroSibilot, G. Pasello, A. Passaro, V. Scotti, P. Solli, E. Stroppa, M. Tiseo, G. Viscardi, L. Voltolini, Y.L. Wu, S. Zai, V. Pancaldi, A. M. Dingemans, J. Van Meerbeeck, F. Barlesi, H. Wakelee, S. Peters, L. Horn, on behalf of the TERAVOLT investigators: COVID-19 in patients with thoracic malignancies (TERAVOLT): first results of an international, registry-based, cohort study. Lancet Oncol. 2045, 30314-30314 (2020)

5. M.M. Montesinos, C.G. Pellizas, Thyroid hormone action on innate immunity. Front. Endocrinol. 10(350), 1-9 (2019)

6. E.L. Jara, N. Muñoz-Durango, C. Llanos, C. Fardella, P.A. González, S.M. Bueno, A.M. Kalergis, C.A. Riedel, Modulating the function of the immune system by thyroid hormones and thyrotropin. Immunol. Lett. 184, 76-83 (2017)

7. S.P. Umland, R.P. Schleimer, S.L. Johnston, Review of the molecular and cellular mechanisms of action of glucocorticoids for use in asthma. Pulm. Pharmacol. Ther. 15, 35-50 (2002)

8. I.J. Elenkov, R.L. Wilder, G.P. Chrousos, E.S. Vizi, The sympathetic nerveean integrative interface between two supersystems: the brain and the immune system. Pharmacol. Rev. 52, 595-638 (2000)
9. T.K. Eisenstein, M.E. Hilburger, Opioid modulation of immune responses: effects on phagocyte and lymphoid cell populations. J. Neuroimmunol. 83, 36-44 (1998)

10. D. Dworakowska, A.B. Grossman, Thyroid disease in the time of COVID-19. Endocrine. 68, 471-474 (2020)

11. B.R. Haugen, E.K. Alexander, K.C. Bible, G.M. Doherty, S.J. Mandel, Y.E. Nikiforov, F. Pacini, G.W. Randolph, A.M. Sawka, M. Schlumberger, K.G. Schuff, S.I. Sherman, J.A. Sosa, D.L. Steward, R.M. Tuttle, L. Wartofsky, 2015 American thyroid association management guidelines for adult patients with thyroid nodules and differentiated thyroid cancer: the American thyroid association guidelines task force on thyroid nodules and differentiated thyroid cancer. Thyroid. 26, 1-133 (2016)

12. A. Tofani, R. Sciuto, R.P. Cioffi, R. Pasqualoni, S. Rea, A. Festa, G.M. Gandolfo, M.C. Arista, C.L. Maini, Radioiodine-induced changes in lymphocyte subsets in patients with differentiated thyroid carcinoma. Eur. J. Nucl. Med. 26, 824-829 (1999)

13. V. Barsegian, S.P. Müller, P.A. Horn, A. Bockisch, A. Lindemann, Lymphocyte function following radioiodine therapy in patients with thyroid carcinoma. Nuklearmedizin. 50, 195-203 (2011)

14. F. Pitoia, F. Jerkovich, Dynamic risk assessment in patients with differentiated thyroid cancer. Endocr Relat Cancer 26, 553-566 (2019)

15. E. Robenshtok, S. Fish, A. Bach, J.M. Domínguez, A. Shaha, R. M. Tuttle, Suspicious cervical lymph nodes detected after thyroidectomy for papillary thyroid cancer usually remain stable over years in properly selected patients. J. Clin. Endocrinol. Metab. 97, 2706-2713 (2012)

16. F.A.B. Schutz, Y. Je, T.K. Choueiri, Hematologic toxicities in cancer patients treated with the multi-tyrosine kinase sorafenib: a meta-analysis of clinical trials. Crit. Rev. Oncol. Hematol. 80, 291-300 (2011)

17. L. Goyal, H. Zheng, M.B. Yurgelun, T.A. Abrams, J.N. Allen, J. M. Cleary, M. Knowles, E. Regan, A. Reardon, A. Khachatryan, R.K. Jain, V. Nardi, D.R. Borger, D.G. Duda, A.X. Zhu, A phase 2 and biomarker study of cabozantinib in patients with advanced cholangiocarcinoma. Cancer. 123, 1979-1988 (2017)

18. S. Nakamichi, H. Nokihara, N. Yamamoto, Y. Yamada, K. Honda, Y. Tamura, H. Wakui, T. Sasaki, W. Yusa, K. Fujino, T. Tamura, A phase 1 study of lenvatinib, multiple receptor tyrosine kinase inhibitor, in Japanese patients with advanced solid tumors. Cancer Chemother. Pharmacol. 76, 1153-1161 (2015)

19. A.R. Filippi, E. Russi, S.M. Magrini, R. Corvò, Covid-19 outbreak In Northern Italy: first practical indications for radiotherapy departments. Int. J. Radiat. Oncol. Biol. Phys. 107(3), 597-599 (2020) 